\title{
Physical Insight into Dispersionless FBG Designs
}

\author{
Michalis N. Zervas ${ }^{1,2}$ and Michael K. Durkin ${ }^{2}$ \\ (1) Optoelectronics Research Centre, University of Southampton, Southampton, UK \\ (2) SPI Lasers, Southampton, UK \\ (mnz@orc.soton.ac.uk)
}

\begin{abstract}
We provide physical insight into the role different sections play in inverse-scatteringdesigned dispersionless FBGs. Using this knowledge we design and fabricate strong $(>30 \mathrm{~dB})$ bidirectional dispersionless filters.

OCIS codes: (050.2770) Gratings; (060.3735) Fiber Bragg gratings; (230.1480) Bragg reflectors; (260.2030) Dispersion;
\end{abstract}

\section{Introduction}

Inverse scattering (IS) has been established as the main design algorithm for advanced grating-based components [1],[2]. Starting from the required transfer function and applying causality, the required refractive-index modulation and chirp profile are obtained. The freedom to choose arbitrarily the response though comes at the expense of device complexity. IS usually results in intricate designs that depart considerably from established intuitive and straightforward approaches. A typical example is the square, dispersionless grating that enables dense WDM and large number of concatenated add-drop functions without substantial penalty [3]. However, such gratings are unidirectional and cannot be used from both sides, which severely compromises their use as add AND drop multiplexers.

So far no attempt has been made to physically understand how these mathematically rigorous designs really work. In this paper, we demonstrate that dispersionless square filters are actually dispersion compensated devices and we physically identify the spatially separate main (dispersive) reflector and dispersion compensator sections. We then use this knowledge to design strong bidirectional gratings with dispersionless response from both sides.

\section{Dispersionless FBGs}

Figure 1(a) shows the refractive index (RI) modulation profile for a square 50GHz dispersionless FBG filter with $R=99.9 \%$. The profile is highly asymmetric and it consists of a main lobe (II) preceded and followed by secondary lobe series I \& III of different height and periodicity. Figure 1(b) shows the reflectivity and transmissivity spectra, while Figure 1(c) shows the reflection group delay from left and right. It clearly shows that the filter is dispersionless only when light enters from the left. The reflection from the opposite side, on the other hand, is highly dispersive. This is known to severely compromises the simultaneous add and drop function of the same FBG.
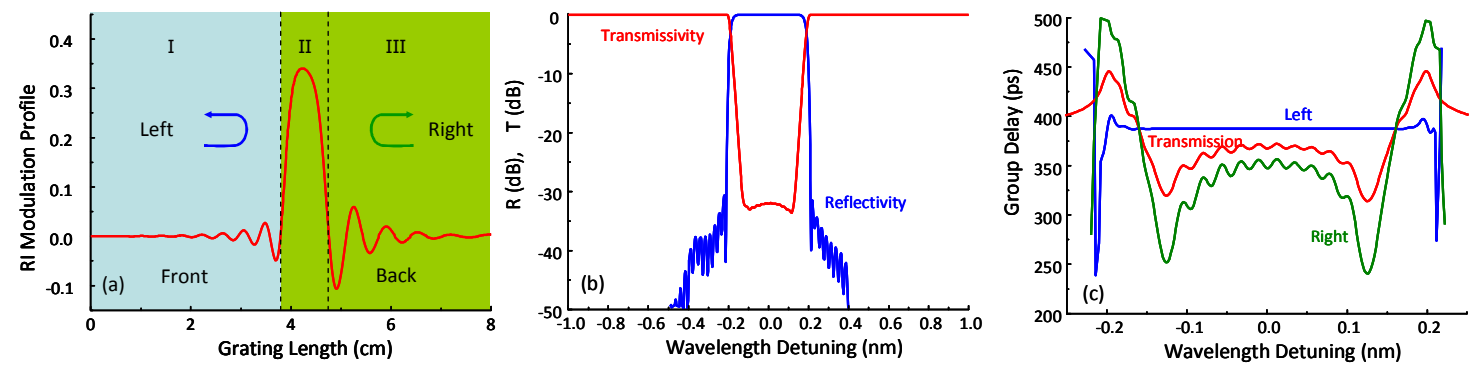

Figure 1: (a) RI Modulation profile (b) Reflectivity \& Transmissivity and (c) Group Delays

The RI modulation profile can be divided into to distinct sections, denoted as front and back parts in Figure 1(a). The front part comprises only the preceding lobe series (section I). The back part on the other hand comprises the main lobe (section II) and the following lobe series (section III). Three different gratings (front-part, back part and whole FBG) were written using a modified "step-and-write" technique [4] and measured separately. Figure 2(a) shows the reflectivity and reflection group delay of the back part alone. This part provides the reflection and it is highly dispersive. Figure 2(b) shows the reflectivity and group delay of the front part alone. The front part provides negligible reflection and it is again highly dispersive with dispersion, however, of opposite sign to the front part. Figure 2(c) finally shows the reflectivity and group delay of the whole dispersionless FBG. Its reflectivity is largely the same with the one of the front part while its group delay is substantially flattened. The residual ripple is of small enough magnitude and period to incur any substantial penalty to high bit-rate data [6]. 

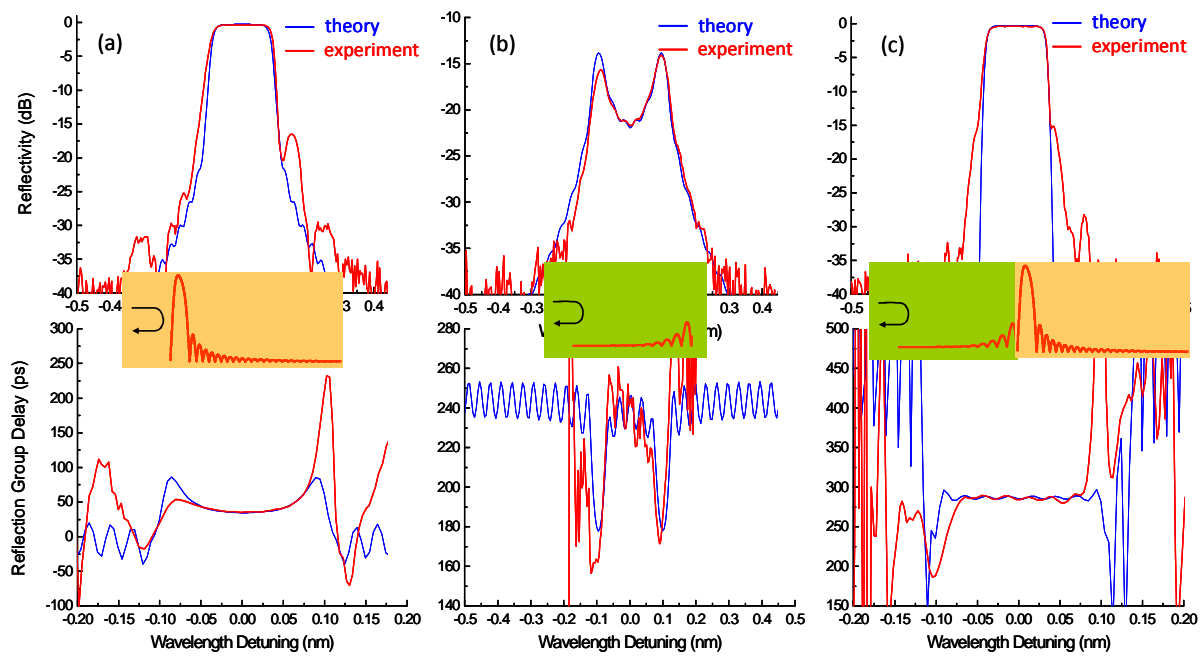

Figure 2: Inverse scattering dispersionless FBGs; (a) main reflector, (b) dispersion compensator and (c) entire grating

\section{Bidirectional Dispersionless FBG Designs}

This physical insight has enabled us to design and fabricate for the first time high reflectivity (>30dB) bidirectional, dispersionless FBGs by modifying exact IS profiles. To achieve this we simply replace section III on the back side with dispersion-compensating section I and symmetrize main lobe I. The symmetric profile is shown in Figure 3(a). It should be stressed that these IS-inspired symmetric profiles are considerably different to sinc profiles, which are are known to provide bidirectional dispersionless response only at low reflectivities $(<\sim 10 \%)$.
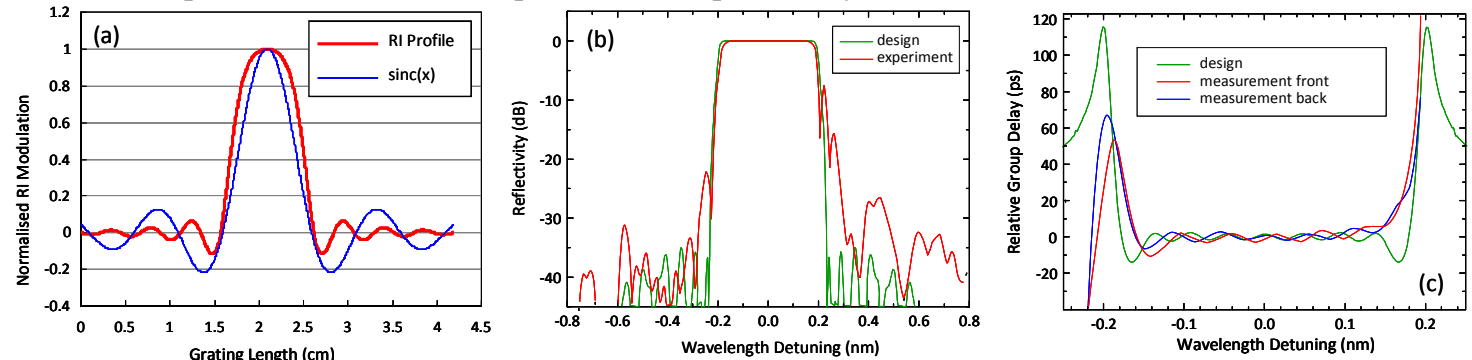

Figure 3: Symmetric low dispersion bidirectional FBG filter (a) RI modulation profile (b) reflectivity and time delay from left and right.

Figure 3(b) and (c) show the corresponding design and experimentally obtained reflectivities and the group delays. It is shown that our symmetric design gives almost identical dispersionless response from both sides, in excellent agreement with theory. More data will be presented at the conference.

\section{Conclusions}

We have shown that IS designed dispersionless square filters are actually dispersion compensated devices and identified the spatially distinct main (dispersive) reflector and dispersion compensator sections. We have used this knowledge to design and fabricate for the first time strong $(>30 \mathrm{~dB})$ bidirectional gratings with dispersionless response from both sides, suitable for simultaneous add AND drop functions out of the same device.

\section{References}

[1] R. Feced, M.N. Zervas, M.A. Muriel, "An efficient inverse scattering algorithm for the design of nonuniform fiber Bragg gratings" J. Quantum Electron. 35, 1105 (1999).

[2] J. Skaar, L.Wang, and T. Erdogan, "On the synthesis of fiber Bragg gratings by layer peeling” J. Quantum Electron, 37, 165-173 (2001).

[3] K. H. Ylä-Jarkko, M. N. Zervas, M. K. Durkin, I. Barry, A. B. Grudinin, "Power Penalties Due to In-Band and Out-of-Band Dispersion in FBG Cascades", J. Lightwave Technol. Vol. 21, 506 (2003).

[4] M.J. Cole, W.H. Loh, R.I. Laming, M.N. Zervas, S. Barcelos “"Moving fibre/phase mask-scanning beam technique for enhanced flexibility in producing fibre gratings with a uniform phase mask", Electron. Lett., vol. 31, pp. 92-94 (1995).

[5] W.H. Loh, M.J. Cole, M.N. Zervas and R.I. Laming, "Compensation of imperfect phase mask with moving fibre-scanning beam technique for production of fibre gratings", Electron. Lett., vol. 31, pp. 1483-1485 (1995).

[6] C. Riziotis, M.N. Zervas, "Effect of in-band group delay ripple on WDM filter performance", 27 ECOC, vol. 4, pp. 492-493 (2001). 